\title{
Energy-Momentum Cathodoluminescence Imaging of Anisotropic Directionality in Elliptical Aluminum Plasmonic Bullseye Antennas
}

\author{
Toon Coenen ${ }^{*},+\oplus \odot$ and Albert Polman ${ }^{\dagger}$ \\ †AMOLF, Center for Nanophotonics, Science Park 104, 1098 XG, Amsterdam, The Netherlands \\ ${ }^{\ddagger}$ Delmic B. V., Kanaalweg 4, 2628 EB, Delft, The Netherlands
}

\section{Supporting Information}

ABSTRACT: We show that geometric eccentricity can be used to strongly tailor the angular radiation profiles of aluminum plasmonic bullseye antennas. High-resolution energy/momentum maps are recorded using a novel cathodoluminescence Fourier imaging technique. The angular profiles for elliptical bullseyes (ellipticity $e=$ $0,0.6$, and 0.8 ) are well described by a $2 \mathrm{D}$ dipole scattering model in which the phase and amplitude of the scattering from the bullseye grooves dictates the angular profile at a given energy. We show that geometric eccentricity is an important parameter to control the radiation profile of bullseye antennas. The new energy-momentum cathodoluminescence imaging technique can be used to map the optical properties of a wide range of dispersive and anisotropic systems, paving the way for a broad range of studies on complex nanophotonic systems.

KEYWORDS: plasmonics, nanoantennas, cathodoluminescence, spectroscopy, aluminum, bullseyes

M anaging the generation, transport, and emission of light at the nanoscale is becoming increasingly important for a large range applications, including solid-state lighting, photovoltaics, optical computing, and sensing. Plasmonic nanoantennas are highly interesting in this context, due to their ability to strongly confine, scatter, and direct light. ${ }^{1-4}$ The plasmonic bullseye antenna, a concentric plasmonic grating system, presents such a highly versatile antenna platform. These antennas are typically driven by a localized source such as a scattering nanoaperture or dye molecule that excite propagating surface plasmon polaritons (SPPs) which subsequently couple out through the bullseye grooves. This scattering process and the resulting optical properties can be controlled by the geometry of the bullseye. In particular, various (derivative) designs have been used for strongly beaming light, ${ }^{5-9}$ local temperature control, ${ }^{10}$ altering polarization, ${ }^{11-15}$ and generation of beams with orbital angular momentum. ${ }^{15-17}$ Indeed, the ability to strongly direct light into a particular direction is a key property of bullseye antennas. So far, research on bullseye antennas has focused mostly on cylindrically symmetric geometries, leaving a key parameter, geometric anisotropy, unexplored. Yet, it is well-known that geometric anisotropy provides a unique way to tailor the emitted light. In particular, it allows to go beyond the azimuthally symmetric response of a circular bullseye, and to engineer anisotropy in energy and momentum space, extending the applicability of bullseye antenna systems in nanophotonics.

In this Article, we investigate the effect of geometric eccentricity on the optical properties of aluminum plasmonic bullseye antennas. Aluminum is a relatively inexpensive, abundant material that is compatible with a large range of fabrication processes. ${ }^{18}$ It has a broadband plasmonic response that extends into the deep-UV, with optical properties that are superior to that of gold in the blue/UV region. Compared to silver, the plasmonic response extends further into the UV, and the chemical stability is better due to the protective native oxide layer. These benefits make it an ideal material for bullseye antennas, enabling emission tuning over a broad range of wavelengths.

We use coherent cathodoluminescence (CL) imaging spectroscopy, a technique in which an electron beam is used to locally polarize the antenna. CL presents an excellent technique for studying plasmonic materials owing to its high-spatial resolution, sensitivity to the local optical environment, and spectrally broadband excitation, ${ }^{19,20}$ and the possibility to obtain angular, ${ }^{21-26}$ polarization, ${ }^{14,27-29}$ and time-resolved information. ${ }^{30-33}$ It has been used to unlock the local resonant behavior and directionality in a large variety of plasmonic antenna systems. ${ }^{14,22,25,26,34}$

Bullseye antennas are composed of diffractive elements (grooves) and are intrinsically highly dispersive, making it challenging to properly track their directionality as a function of wavelength. Additionally, in the case of elliptical bullseyes, the response is highly anisotropic in momentum space. As such, the response has to be measured for a large range of azimuthal angles to obtain a full understanding of the optical properties. Previously, angle-resolved CL spectroscopy and polarimetry ${ }^{14}$

Received: December 13, 2018

Published: December 26, 2018 

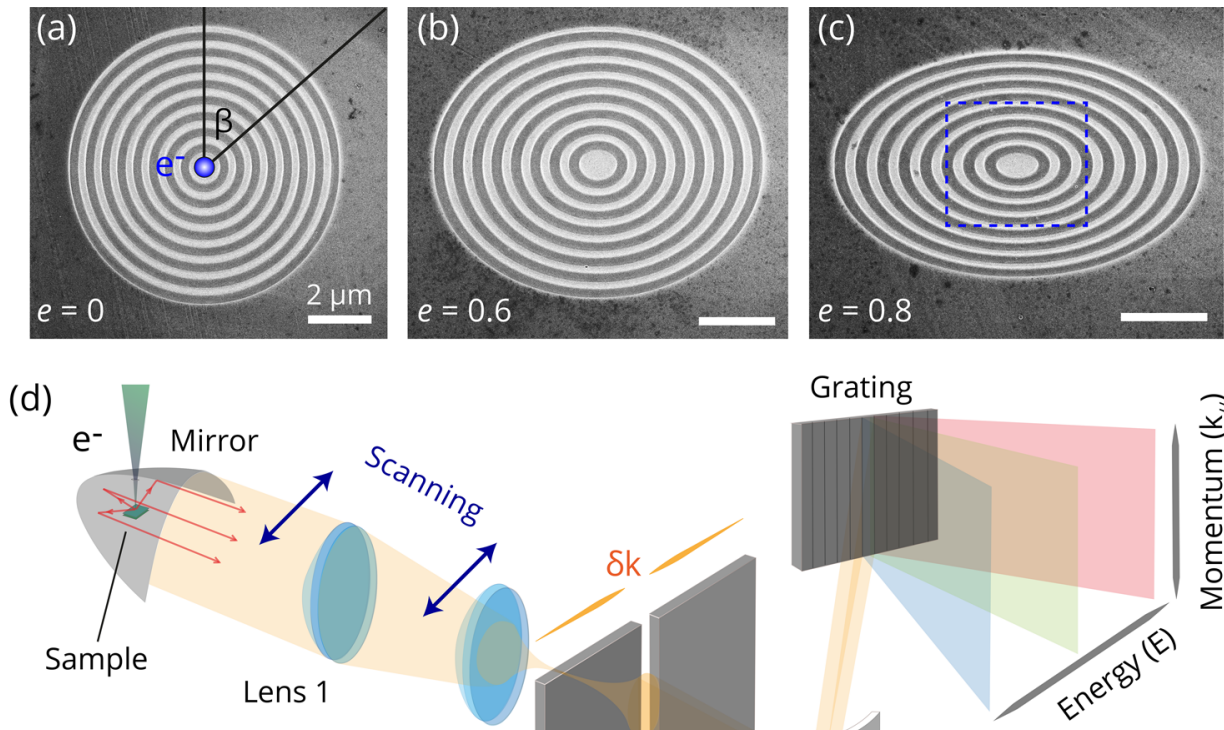

Lens 2
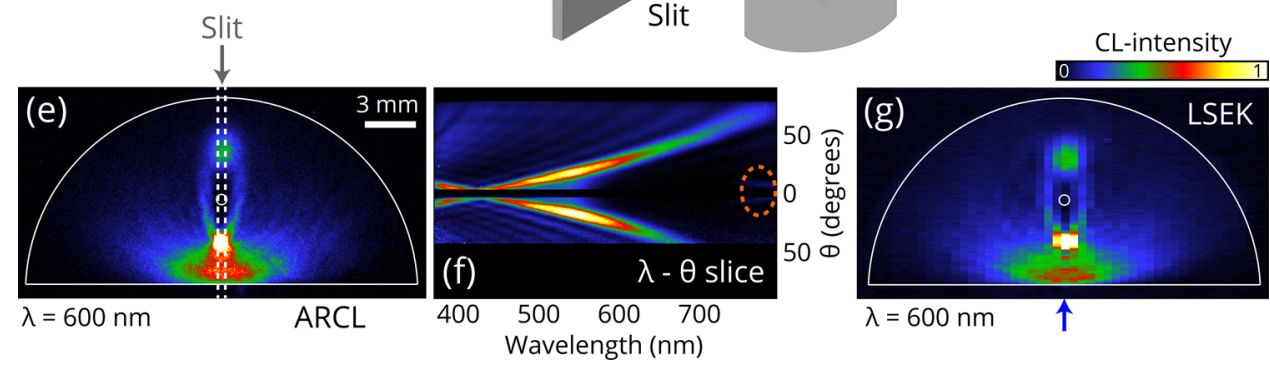

Figure 1. SEM images of aluminum bullseye antennas with (a) $e=0,(\mathrm{~b}) e=0.6$, and (c) $e=0.8$. In (a), the electron beam excitation position (blue dot) and angle $\beta$ are schematically shown. (d) Schematic overview of the experimental setup. The blue arrows indicate the lens movement for LSEK imaging. The grating is placed on a turret and can be readily interchanged for an aluminum mirror for ARCL. (e) Raw ARCL CCD image acquired using a $\lambda=600 \mathrm{~nm}$ band-pass filter with a $40 \mathrm{~nm}$ bandwidth. The position of the slit in the spectrograph is indicated by the white dashed lines. The solid white line shows the parabolic mirror outline. (f) Raw $\lambda-\theta$ map, corresponding to the slice acquired at the center of the paraboloid mirror. The orange dashed ellipse indicates the second grating order of the spectrograph diffraction grating. (g) Raw composite LSEK image acquired by taking multiple $E$ $-k$ acquisitions for different lens positions. The image corresponds to a center wavelength of $600 \mathrm{~nm}$ with a $17 \mathrm{~nm}$ bandwidth. The blue arrow indicates the $\lambda-\theta$ slice shown in (f).

were performed by projecting the emitted light on a CCD imaging camera, with spectral sensitivity achieved using broadband bandpass filters. While this enabled momentum spectroscopy, the lack of high spectral sensitivity is a major drawback in the investigation of dispersive antenna radiation profiles. Recently, we codeveloped the energy-momentum CL imaging technique with a much higher energy resolution, where momentum was probed along a single plane. ${ }^{35}$ Here, we extend this technique with a scanning optical geometry, which we use to scan the Fourier pattern across the input slit of a spectrograph, enabling the collection of two-dimensional momentum maps. This approach enables Fourier microscopy over an octave of bandwidth and a large numerical aperture (NA), with high momentum and energy resolution. We demonstrate that geometric eccentricity provides is powerful parameter to control the directionality of plasmonic bullseye antennas.

\section{RESULTS AND DISCUSSION}

Fabrication. We fabricated three sets of bullseye gratings with varying eccentricities $(e)$ in a Czochralski-grown singlecrystal aluminum substrate, using focused-ion-beam milling (Thermo Fisher/FEI Helios 450 dual beam) with a $30 \mathrm{kV}$ gallium ion beam and a 28 pA beam current. In particular, we fabricated bullseyes with $e=0$ (circular bullseye), 0.6 , and 0.8 , all with a groove pitch $a$ of $500 \mathrm{~nm}$ along the major axis and 500, 400 , and $300 \mathrm{~nm}$ along the minor axis, respectively. The central plateau in the bullseye has a diameter of $2 a$ and is surrounded by a concentric grating with a 50/50 duty cycle consisting of 8 grooves $($ depth $=130 \mathrm{~nm})$. Scanning electron micrographs of the resulting structures are shown in Figure $1 \mathrm{a}-\mathrm{c}$.

Setup. For the CL experiments we use a Delmic SPARC CL detection system, mounted on a Thermo Fisher 650 Quanta field-emission gun scanning electron microscope (SEM). The CL-system is equipped with a $2048 \times 512$ pixel back-illuminated CCD array mounted on a Czerny-Turner spectrograph. The light is collected with an aluminum paraboloid mirror $(1.47 \pi$ sr acceptance angle), which is aligned with respect to the electron beam and sample using a motorized micropositioning stage inside of the vacuum chamber. The spectrograph contains a motorized entrance slit, a filter wheel with band-pass color filters, and a turret with two pieces of optics on opposite sides. It has a 300 lines $/ \mathrm{mm}$ ruled grating, blazed for a free space wavelength $\lambda=500 \mathrm{~nm}$ on one side and a flat aluminum mirror on the other. A schematic overview of the experimental setup is shown in Figure 1d. In this work we employ two complementary 

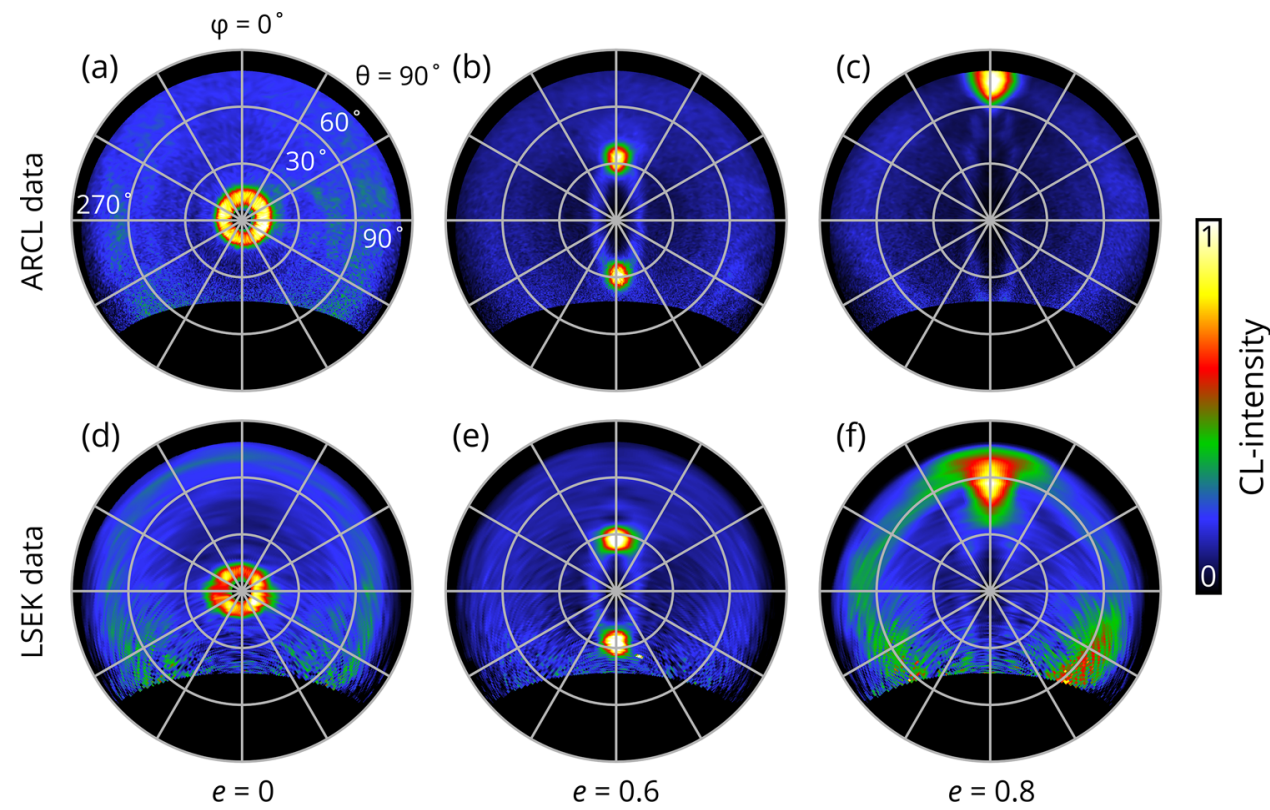

Figure 2. Angular profiles for the three bullseyes, with $e=0,0.6$, and 0.8 (minor axis aligned with the slit), as measured with conventional single-shot ARCL $(a-c)$ and LSEK imaging $(\mathrm{d}-\mathrm{f})$ for $\lambda=600 \mathrm{~nm}$. Each column in the figure represents a different eccentricity as noted below.

Fourier imaging modes: (1) In both approaches we project the Fourier $(k$-space) pattern on the entrance slit of the spectrograph. When the slit is opened completely $(15 \mathrm{~mm})$ and the aluminum mirror is selected on the turret in the spectrograph, we can acquire a full angular pattern/momentum distribution within the NA of the paraboloid collection optics by projecting the angular distribution onto the CCD camera. The CL emission intensity is measured for a large range of zenithal $(\theta)$ and azimuthal $(\varphi)$ angles simultaneously. Wavelength specificity is attained with the band-pass filters in the filter wheel in this case (see references ${ }^{21,22}$ for more details on this approach). We will refer to this imaging mode as angle-resolved CL (ARCL). A representative raw ARCL image, acquired on a $e=0.6$ bullseye is shown in Figure 1e. Because this technique has been successfully applied to many nanophotonic systems ${ }^{2,34,36}$ it provides a good benchmark for CL Fourier imaging. (2) In the second approach, the entrance slit is closed such that a narrow opening remains with an adjustable width of $10-2500 \mu \mathrm{m}$. The slit acts as a filter in angular/momentum space and normally selects the central part of the Fourier pattern corresponding to the center of the paraboloid as indicated in Figure $1 \mathrm{~d}$. In particular, a range of zenithal emission angles is collected for a fixed azimuthal angle $\left(\varphi=0^{\circ}, 180^{\circ}\right)$ (see ref 35 for more information). This selection of the angular emission pattern is sent to the diffraction grating which decomposes the light into different wavelengths, leading to a hybrid 2D CCD image with $\lambda$ on the horizontal axis and $\theta$ on the vertical axis. An example of such a map is shown in Figure 1f. This $\lambda-\theta$ map can directly be converted into an energy $(E)$ momentum $(k)$ map by applying the appropriate coordinate transform. ${ }^{35}$ We will refer to this method as $E-k$ imaging. The combination of CCD array size and broadband diffraction grating employed here results in a spectral range spanning more than one optical octave, giving rise to a second diffraction order from the spectrograph grating in the low energy range of the $E-$ $k$ maps (see orange dashed circles in Figures If and $3 a$ ). In section 1 in the Supporting Information, this is discussed in more detail. We note that both $E-k$ imaging and energy-real space $(E-x)$ imaging are performed in a similar manner in optical microscopy systems. ${ }^{37-39}$

Although the $E-k$ imaging method is superior to the ARCL method in terms of energy resolution, it only partially maps kspace along a specific range of zenithal angles (whereas a "full" $k$ space image is obtained in ARCL. Here, we lift this limitation by extending the $E-k$ technique with scanning lens optics. In particular, we scan the Fourier pattern across the slit, by transversely moving two motorized incoupling lenses that are placed in front of the entrance slit (see Figure 1d). The projected Fourier pattern in the entrance-slit plane is $7.6 \mathrm{~mm}$ wide and the lenses are placed $\sim 15 \mathrm{~cm}$ away from the slit plane. The displacement of the Fourier pattern during the lens-movement corresponds to a change in angle of $<1.5^{\circ}$ with respect to the slit plane which has a negligibly small effect on the overall pattern. Therefore, we can safely assume that the Fourier pattern is only displaced and not distorted when the lenses are moved.

To obtain a full 3D $E-k$ image, the lenses are first moved outward such that Fourier pattern falls just outside the entrance slit. Subsequently, they move in the opposite direction such that an entire pattern crosses the slit, while $E-k$ images are acquired with the camera. The displacement of the Fourier pattern is linear with the lens movement in this geometry, simplifying the approach. The entrance slit width is matched to the step size of the Fourier pattern movement to prevent under/oversampling. For plasmonic systems such as the bullseyes studied here, a slit width of $150-200 \mu \mathrm{m}$ presents a good compromise between signal level, acquisition time, and angular resolution. These widths correspond to $\sim 40-60 E-k$ pattern acquisitions. We correct for sample drift by imaging a reference region every $30 \mathrm{~s}$, with the secondary electron detector in the SEM. We will refer to this method as lens-scanning energy-momentum (LSEK) imaging. Figure $1 \mathrm{~g}$ shows a wavelength slice through a raw LSEK scan, acquired with a $150 \mu \mathrm{m}$ slit width and 57 lens positions.

Figure $2 \mathrm{a}-\mathrm{c}$ shows three angular profiles acquired with the ARCL approach on three bullseyes with $e=0,0.6$, and 0.8 , respectively, with the short axis of the bullseye parallel to the slit 

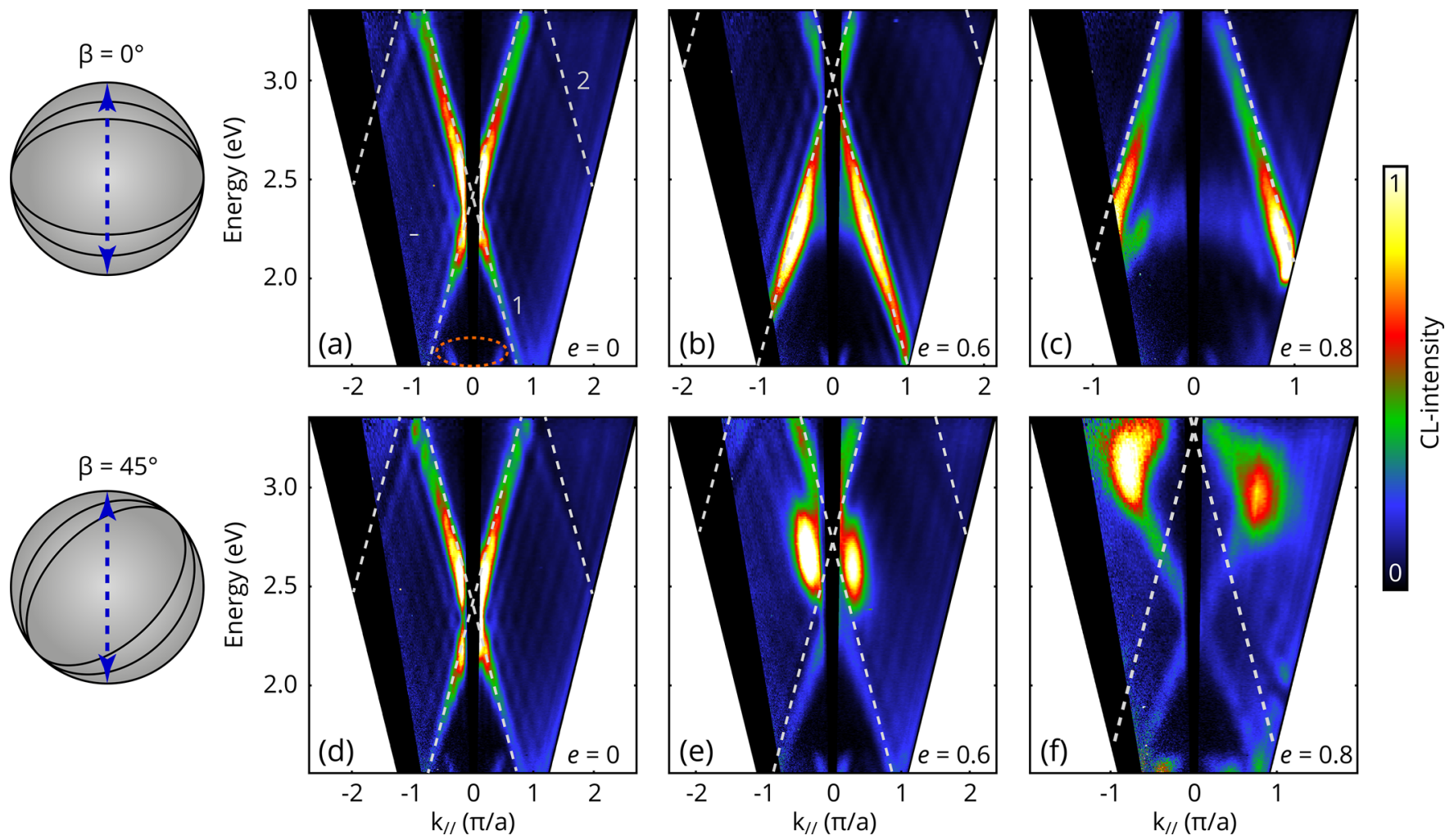

Figure 3. $E-k$ maps acquired on bullseyes with $(\mathrm{a}, \mathrm{d}) e=0,(\mathrm{~b}, \mathrm{e}) e=0.6$, and $(\mathrm{c}, \mathrm{f}) e=0.8$ for $\beta=0^{\circ}$ and $45^{\circ}$. The orientation of the bullseye structures with respect to the entrance slit (blue arrow) is shown schematically on the left. The light gray dashed lines indicate the calculated dispersion based on a 1D SPP grating model. In (a), the different bullseye diffraction orders $(n)$ are indicated. The orange dashed ellipse indicates the second grating order of the spectrograph grating.

$\left(\varphi=0^{\circ}\right)$. A summary of the acquisitions parameters is given in Table S1 in the Supporting Information. The electron beam was positioned in the center of the plateau, as indicated in Figure 1a, and a $\lambda=600 \mathrm{~nm}$ color filter (40 nm bandwidth) was used. For the circular bullseye, we observe a directional doughnut beam, which is expected when the bullseye is symmetrically excited in the center. ${ }^{14,36}$

For the elliptical bullseyes two intense spots are observed along the short axis of the ellipse. As the eccentricity increases ( $a$ decreases along minor axis) these spots move progressively toward higher angles. LSEK images are shown in Figure $2 \mathrm{~d}-\mathrm{f}$ for $\lambda=600 \mathrm{~nm}$. They are composed of $57 E-k$ slices with a $\Delta \lambda=$ $1.8 \mathrm{~nm}$ bandwidth, more than a factor 20 smaller than the typical bandwidth used in the ARCL. Nevertheless, the LSEK patterns match well with the ARCL patterns demonstrating that this technique can be used to accurately measure angular profiles with high energy resolution. A full $3 \mathrm{D}$ visualization of the $3 \mathrm{D}$ LSEK data set in which the continuous change of the angular profile can be seen as a function of wavelength/energy is included in Supporting Information, section 3.

To investigate the bullseye dispersion in more detail we study the CL emission in energy-momentum space along three different angles of the bullseye's minor axis relative to the slit $(\beta$ $\left.=0^{\circ}, 45^{\circ}, 90^{\circ}\right)$. The angle is set using the SEM stage rotation. A detailed discussion on this approach is given in Supporting Information, section 4. Additionally, we have included a discussion of how the different angle-resolved CL approaches compare (Supporting Information, section 5).

Data for $\beta=0^{\circ}$ and $45^{\circ}$ are shown in Figure 3a-f. Maps for $\beta$ $=90^{\circ}$ are shown in Figure S1a-c in the Supporting Information. The color maps show the CL intensity as a function of photon energy and normalized parallel momentum $k_{\|}\left(k_{0} \sin (\theta) a / \pi\right)$ within the light cone. The black band on the $k_{\|}<0$ side corresponds to the open side of the paraboloid mirror where light is not collected. In all the maps we see two main emission bands which correspond to diffraction from the bullseye grating. As expected, the maps corresponding to $\beta=0^{\circ}$ and $45^{\circ}$ are very similar for the circular $(e=0)$ bullseye. The bullseye is driven by a concentric SPP wave that is generated in the center of the bullseye. This bound SPP wave is coupled to free-space radiation by diffraction from the bullseye grooves. The diffraction of the SPPs from the grooves can be approximated with a $1 \mathrm{D}$ grating model, taking into account the SPP dispersion on aluminum. Here, $k_{\|}=\left[\operatorname{Re}\left(k_{\mathrm{SPP}}\right)-2 n \pi / a\right] * a / \pi$, where $n$ is an integer corresponding to the diffraction order (light gray dashed lines). For the combinations of photon energies and groove pitches considered here, the bullseyes emit light in $n=1,2$ diffraction orders as indicated in Figure 3a. Due to the mirror symmetry of the excitation and structure these lead to two pairs of symmetrical emission bands in the $E-k$ map. Along the short (Figure $3 a-c$ ) and long axes (Figure $\mathrm{S} 1 \mathrm{a}-\mathrm{c}$ ) of the bullseyes, this model matches well with the data.

The reduction in $a$ along the short axis $\left(\beta=0^{\circ}\right)$ for increasing eccentricity leads to a blueshift of the diffraction orders, which is clearly visible in the corresponding $E-k$ maps. This is consistent with the movement of the emission lobes toward higher polar angle for a given energy, as observed in Figure 2b,c. For $\beta=45^{\circ}$, this blueshift of the dispersion is also observed, but it is less pronounced because the differences in $a$ are smaller along this axis. For $\beta=90^{\circ}$, no shift is present, as $a$ is equal for all eccentricities. The exact intensity distributions along the bands differ though (see Figure S1). Because the pitch is the same this 

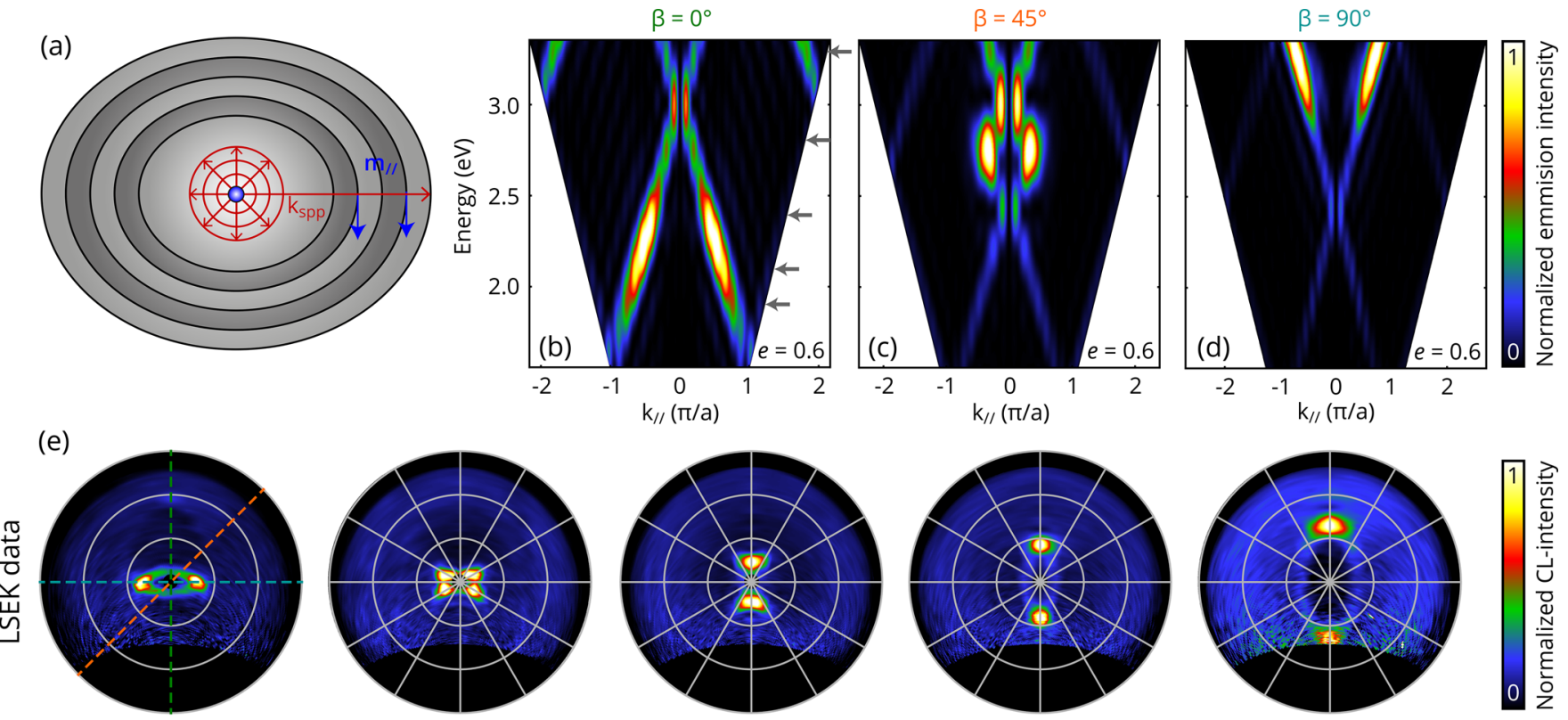

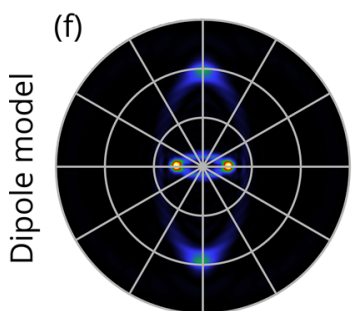

375 nm, $3.31 \mathrm{eV}$

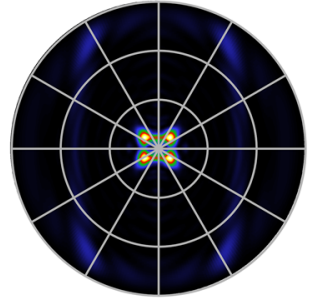

445 nm, $2.79 \mathrm{eV}$

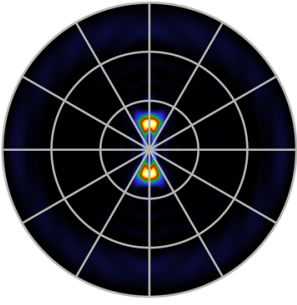

$515 \mathrm{~nm}, 2.41 \mathrm{eV}$

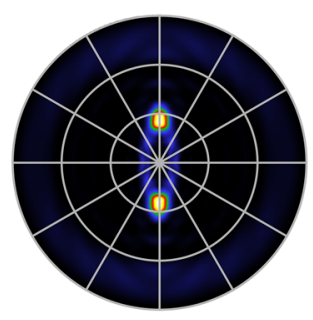

585 nm, $2.12 \mathrm{eV}$

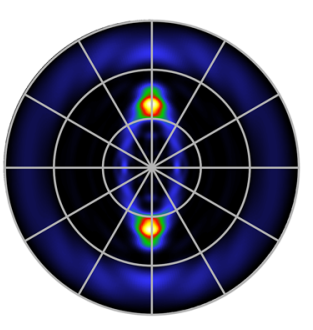

655 nm, 1.89 eV

Figure 4. (a) Schematic representation of the dipole model where the blue arrows represent the magnetic dipole positions and orientations and the red arrows represent the plasmon wave that is excited in the bullseye center. Calculated $E-k$ maps for (b) $\beta=0^{\circ}$, (c) $\beta=45^{\circ}$, (d) $\beta=90^{\circ}$, for $e=0.6$. (e) Five angular patterns as measured with LSEK imaging and (f) corresponding patterns as calculated with the dipole model. The corresponding wavelengths/energies are listed below. For reference these energies are also indicated by the gray arrows in (b). The three $\beta$ angles for (b) $-(d)$ are indicated by the colored dashed lines in the left angular pattern in (e).

change in intensity distribution must come from the change in eccentricity. Next to the bands, a weaker complex interference pattern is visible. Characteristic cross cuts and a discussion on the line widths of the bands are given in Figure S2 and Supporting Information, section 7, respectively. The effect of the number of grooves is also discussed.

Interestingly, a dark line is visible close to where the $n=2$ grating order is located some of the $E-k$ maps (most visible in Figure $3 a, d$ ). Such features have been observed before in bullseyes $^{12-14}$ and could be related to inference of different dipole components in the plasmon groove scattering and interference of the bullseye emission with the direct transition radiation coming from the electron beam impact position.

As seen in Figure $3 \mathrm{e}, \mathrm{f}$, for $\beta=45^{\circ}$ and $e>0$, the agreement with the $1 \mathrm{D}$ grating model breaks down. The emission bands no longer follow the calculated grating order dispersion. Furthermore, we observe bright spots in the maps at particular points in the $E-k$ space, which move with eccentricity and also cannot be explained by a 1D SPP grating model. The reason for this mismatch is that the resulting patterns in $E-k$ space are, in fact, governed by the coherent scattering of the grooves in 2D. To include the $2 \mathrm{D}$ character of the bullseye emission we take the following approach. SPPs are transverse-magnetic waves and it is known that they can drive a magnetic dipole resonance in the groove where a (partial) current loop is induced in the groove edges. $^{34,40-42}$ As has been shown before, (composite) plasmonic systems can be approximated as a collection of point scatterers. ${ }^{36,43,44}$ In particular, the bullseye emission can be described as a collection of in-plane magnetic dipoles $\left(m_{\|}\right)$, where the phase and amplitude are dictated by the incoming SPP wave. ${ }^{13,41}$ For the $m_{\|}$far-field we use the formalism as described in ref 45 , where we assume that the dipoles are positioned $10 \mathrm{~nm}$ above the top surface of the bullseye, in the center of each grating period as schematically illustrated in Figure 4a. Details of the model are discussed in the Supporting Information, section 8 .

We employ this 2D dipole model to calculate both angular distributions and $E-k$ maps. Figure $4 \mathrm{~b}-\mathrm{d}$ shows theoretical $E$ $-k$ maps for a $e=0.6$ bullseye at $\beta=0^{\circ}, 45^{\circ}$, and $90^{\circ}$. These match remarkably well with the corresponding experimental maps in Figure $3 \mathrm{~b}\left(\beta=0^{\circ}\right)$, Figure $3 \mathrm{e}\left(\beta=45^{\circ}\right)$, and Figure S1b $\left(\beta=90^{\circ}\right)$. For the orthogonal directions $\beta=0$ and $90^{\circ}$, the lobes follow the 1D SPP grating dispersion, shifting to shorter wavelength as the grating pitch is increased. For $\beta=45^{\circ}$, the more complex emission lobe pattern observed in Figure $3 \mathrm{e}$ is reproduced well in the model. Furthermore, in Figures S1 and S4, experimental and calculated $E-k$ maps are shown for $\beta=$ $90^{\circ}, e=0,0.6,0.8$ and for $e=0.8, \beta=0^{\circ}$ and $45^{\circ}$, respectively, where the calculations also match well with the data. The fact that the band placement, width, intensity distribution, and fine structure are very similar, even for $\beta=45^{\circ}$, where the $1 \mathrm{D}$ model breaks down, demonstrates that this model is a good 

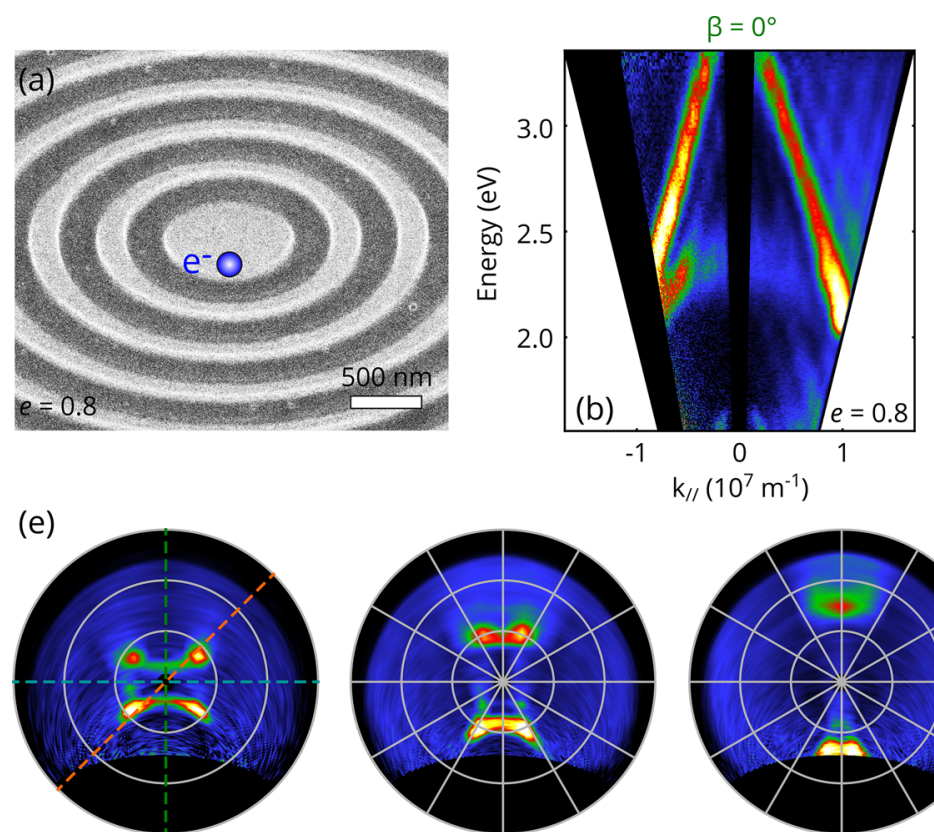

375 nm, $3.31 \mathrm{eV}$

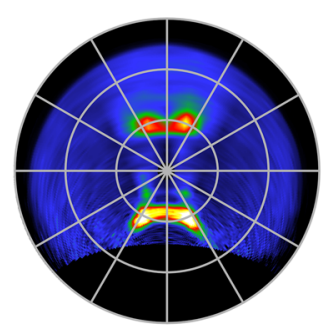

$445 \mathrm{~nm}, 2.79 \mathrm{eV}$

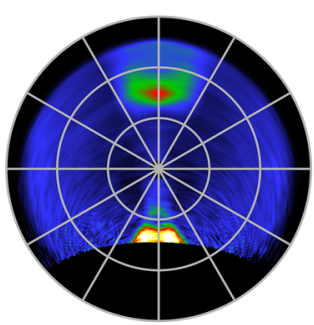

$515 \mathrm{~nm}, 2.41 \mathrm{eV}$
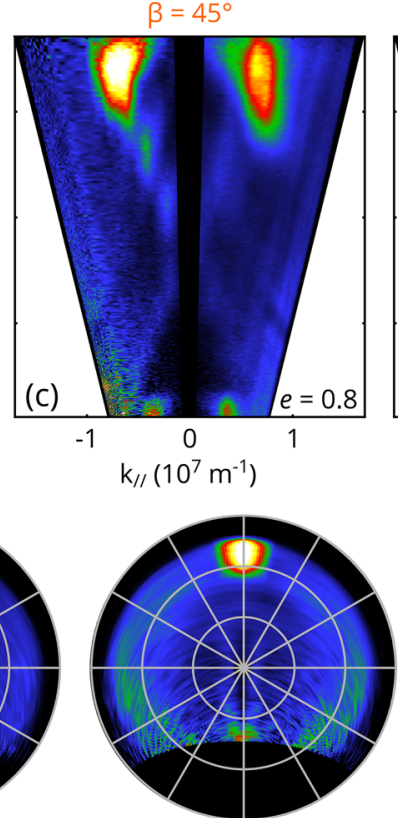

585 nm, 2.12 eV

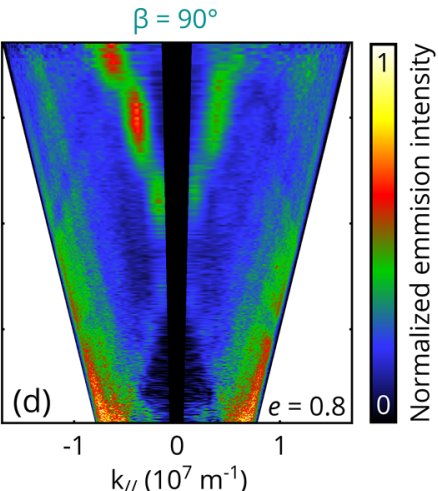

Figure 5. (a) SEM micrograph of the central section of a $e=0.8$ bullseye, as indicated by the blue dashed box in Figure $1 c$. The blue dot shows the electron beam impact position. Corresponding $E-k$ maps for (b) $\beta=0^{\circ}$, (c) $\beta=45^{\circ}$, (d) $\beta=90^{\circ}$, as measured using the LSEK approach. (e) Five angular patterns for five different energies, extracted from the data set. In the left pattern in (e) the different $\beta$ angles as shown in (b) $-(\mathrm{d})$ are indicated with a color code dashed line.

approximation to the bullseye. The data confirm that the observed patterns indeed result from a coherent $2 \mathrm{D}$ interference between the grooves, where the phase and amplitude of the SPP wave at the groove positions play a critical role in determining the overall pattern in $E-k$ space.

Next, in Figure 4e,f we compare the theoretical and experimental full angular distributions for the $e=0.6$ bullseye for five different wavelengths/energies. Strong agreement is observed between experiment and theory with all main trends reproduced: (1) An angular profile with two strong emission lobes is observed for the highest energy $(3.31 \mathrm{eV})$, with an elliptical halo near the origin seen in both theory and experiment. (2) An angular profile with four strong emission lobes is observed at $2.79 \mathrm{eV}$ for both experiment and theory. These lobes correspond to the bright spots in the $E-k$ map for $\beta$ $=45^{\circ}$ (Figure 4c) and are clearly observed in the $E-k$ map for $\beta$ $=45^{\circ}$ in Figures $3 \mathrm{e}$ and $4 \mathrm{c}$. (3) Decreasing the energy further, angular patterns with two main lobes along $\beta=0^{\circ}$ are observed, in full agreement with the $E-k$ maps for $\beta=0^{\circ}$ in Figures $3 \mathrm{~b}$ and $4 \mathrm{~b}$. The lobe angle increases with decreasing energy, consistent with the grating diffraction model.

The fact that the patterns at 3.3 and $2.4 \mathrm{eV}$ look similar (albeit rotated by $90^{\circ}$ ) confirms that the $k$-space behavior of the elliptical bullseye along the minor and major axis is similar, but shifted in energy. Due to the symmetry of the bullseyes and central excitation position the emission destructively interferes at $k_{\|}=0$ (not visible in experiment due to paraboloid hole), leading to a null in the angular profile as was also observed for radially symmetric antenna sources. ${ }^{14,36}$ In the CL data, the signal at larger $k_{\|}\left(\theta>45^{\circ}\right)$ is higher than in the model; potential causes are described in Supporting Information, section 8.

Finally, we vary the electron beam excitation position on the bullseye structure. Figure 5 shows $E-k$ maps $(b-d)$ and angular profiles (e), as measured with the LSEK approach on a $e$ $=0.8$ bullseye for an off-center excitation position, as indicated in (a). Both the $E-k$ and angular patterns show an asymmetry parallel to the axis on which the electron beam is displaced (the minor ellipse axis in this case), as was observed before in circular plasmonic patch antennas ${ }^{36}$ and bullseyes ${ }^{14}$ as well. Although the patterns are clearly asymmetric, they are not altered drastically. That means that the bullseye response is rather robust against emitter displacements within the central plateau in this case, which could be a benefit in a sensing application, for example.

We have shown that the eccentricity of the bullseye can be used to tailor an anisotropic response in $E-k$ space, that is profoundly different from the conventional azimuthally symmetric circular bullseye emission. The orthogonal beaming behavior for different energies along minor and major axes of the elliptical bullseyes, as shown in Figures 3 and 4 could potentially be utilized for a microscale directional spectrum splitting device. This could be used to separate the emission from a mix of two or more emitter species or to separate different emission bands from a single emitter, for example. By varying the electron beam position, robustness of the directionality can be tested systematically. In this case, the bullseye behavior can be further tailored to yield a more asymmetric directional beam. From CL methodology perspective, the LSEK approach presented here could be combined with the angle-resolved polarimetry approach that has been developed before. ${ }^{14}$ Furthermore, for materials that efficiently emit incoherent $\mathrm{CL}$, such as direct band gap semiconductor materials, the CL yield is orders of magnitude higher than the plasmonic signals studied here, allowing high-resolution LSEK imaging with significantly shorter dwell times and narrower slit widths.

Finally, we envision that this technique can be employed in studies on topological photonics, (quasi) periodic, hyperuniform, and random complex media, metasurfaces, and nanoantennas. 
In conclusion, we have studied the influence of geometric eccentricity on the directionality of aluminum plasmonic bullseye antennas using cathodoluminescence imaging. For this purpose, we use a novel energy-momentum CL imaging technique in which we scan the Fourier pattern over the entrance slit of a spectrograph, allowing us to obtain all momenta collected by the paraboloid mirror. This yields a highresolution energy-momentum data set containing CL data for a large range of energies and momenta. We study the directionality in anisotropic aluminum bullseye antennas and find that the emission along the major and minor axes can be well understood from a 1D grating model. For other axes a more advanced dipole model, taking into account the scattering processes in $2 \mathrm{D}$ describes the data well. The results demonstrate that the directionality in bullseye antennas is strongly tunable with eccentricity. Furthermore, the study establishes the LSEK imaging as a powerful analytical momentum spectroscopy tool in cathodoluminescence studies on nanoscale materials and devices.

\section{ASSOCIATED CONTENT}

\section{S Supporting Information}

The Supporting Information is available free of charge on the ACS Publications website at DOI: 10.1021/acsphotonics.8b01711.

\section{Movie 1 (MPG). \\ Movie 2 (MPG).}

Description of second diffraction order in spectroscopy system, acquisition parameters, descriptions of the LSEK movies (movie 1, movie 2), stage rotation approach, comparison with other ARCL techniques, $E-k$ data and calculations for $\beta=90^{\circ}$, cross cuts through $E-k$ data, effect of the number of grooves on directionality, details on dipole model, comparison CL data, and calculations for $e=0.8$ (PDF).

\section{AUTHOR INFORMATION}

\section{Corresponding Author}

*E-mail: coenen@delmic.com.

\section{ORCID}

Toon Coenen: 0000-0002-8043-9798

\section{Notes}

The authors declare the following competing financial interest(s): T.C. is employee and A.P. is co-founder and coowner of Delmic B.V., a company that has brought a cathodoluminescence system on the market based on a design developed at AMOLF and that was used in this work.

\section{ACKNOWLEDGMENTS}

We would like to acknowledge Prof. Riccardo Sapienza, Sandro Mignuzzi (Imperial College London), Prof. Femius Koenderink, and Dr. Nick Schilder (AMOLF) for useful discussions. Schematic in Figure 1d courtesy of Sandro Mignuzzi. This work is part of the research program of the "Nederlandse organisatie voor Wetenschappelijk Onderzoek" (NWO). It is also funded by the European Research Council (ERC).

\section{REFERENCES}

(1) Atwater, H. A.; Polman, A. Plasmonics for Improved Photovoltaic Devices. Nat. Mater. 2010, 9, 205-213.
(2) Schuller, J. A.; Barnard, E. S.; Cai, W.; Jun, Y. C.; White, J. S.; Brongersma, M. L. Plasmonics for Extreme Light Concentration and Manipulation. Nat. Mater. 2010, 9, 193-204.

(3) Novotny, L.; Van Hulst, N. Antennas for Light. Nat. Photonics 2011, 5, 83-90.

(4) Halas, N. J.; Lal, S.; Chang, W. S.; Link, S.; Nordlander, P. Plasmons in Strongly Coupled Metallic Nanostructures. Chem. Rev. 2011, 111, 3913-3961.

(5) Lezec, H. J.; Degiron, A.; Devaux, E.; Linke, R. A.; Martin-Moreno, L.; Garcia-Vidal, F. J.; Ebbesen, T. W. Beaming Light from a Subwavelength Aperture. Science 2002, 297, 820-822.

(6) Caglayan, H.; Bulu, I.; Ozbay, E. Beaming of Electromagnetic Waves Emitted through a Subwavelength Annular Aperture. J. Opt. Soc. Am. B 2006, 23, 419.

(7) Martín-Moreno, L.; García-Vidal, F. J.; Lezec, H. J.; Degiron, A.; Ebbesen, T. W. Theory of Highly Directional Emission from a Single Subwavelength Aperture Surrounded by Surface Corrugations. Phys. Rev. Lett. 2003, 90, 4.

(8) Aouani, H.; Mahboub, O.; Bonod, N.; Devaux, E.; Popov, E.; Rigneault, H.; Ebbesen, T. W.; Wenger, J. Bright Unidirectional Fluorescence Emission of Molecules in a Nanoaperture with Plasmonic Corrugations. Nano Lett. 2011, 11, 637-644.

(9) Andersen, S. K. H.; Bogdanov, S.; Makarova, O.; Xuan, Y.; Shalaginov, M. Y.; Boltasseva, A.; Bozhevolnyi, S. I.; Shalaev, V. M. Hybrid Plasmonic Bullseye Antennas for Efficient Photon Collection. ACS Photonics 2018, 5, 692-698.

(10) Crick, C. R.; Albella, P.; Ng, B.; Ivanov, A. P.; Roschuk, T.; Cecchini, M. P.; Bresme, F.; Maier, S. A.; Edel, J. B. Precise Attoliter Temperature Control of Nanopore Sensors Using a Nanoplasmonic Bullseye. Nano Lett. 2015, 15, 553-559.

(11) Gorodetski, Y.; Lombard, E.; Drezet, A.; Genet, C.; Ebbesen, T. W. A Perfect Plasmonic Quarter-Wave Plate. Appl. Phys. Lett. 2012, $101,201103$.

(12) Mohtashami, A.; Osorio, C. I.; Koenderink, A. F. Angle-Resolved Polarimetry of Antenna-Mediated Fluorescence. Phys. Rev. Appl. 2015, 4, No. 054014.

(13) Osorio, C. I.; Mohtashami, A.; Koenderink, A. F. K-Space Polarimetry of Bullseye Plasmon Antennas. Sci. Rep. 2015, 5, 9966.

(14) Osorio, C. I.; Coenen, T.; Brenny, B. J. M.; Polman, A.; Koenderink, A. F. Angle-Resolved Cathodoluminescence Imaging Polarimetry. ACS Photonics 2016, 3, 147-154.

(15) Kerber, R. M.; Fitzgerald, J. M.; XIAO, X.; Oh, S. S.; Maier, S.; Giannini, V.; Reiter, D. Interaction of an Archimedean Spiral Structure with Orbital Angular Momentum Light. New J. Phys. 2018, 20, No. 095005.

(16) Gorodetski, Y.; Drezet, A.; Genet, C.; Ebbesen, T. W. Generating Far-Field Orbital Angular Momenta from near-Field Optical Chirality. Phys. Rev. Lett. 2013, 110, 203906.

(17) Röhrich, R.; Hoekmeijer, C.; Osorio, C. I.; Koenderink, A. F. Quantifying Single Plasmonic Nanostructure Far-Fields with Interferometric and Polarimetric k-Space Microscopy. Light: Sci. Appl. 2018, 7,65 .

(18) Knight, M. W.; King, N. S.; Liu, L.; Everitt, H. O.; Nordlander, P.; Halas, N. J. Aluminum for Plasmonics. ACS Nano 2014, 8, 834-840.

(19) García De Abajo, F. J. Optical Excitations in Electron Microscopy. Rev. Mod. Phys. 2010, 82, 209-275.

(20) Coenen, T.; Haegel, N. M. Cathodoluminescence for the 21st Century: Learning More from Light. Appl. Phys. Rev. 2017, 4, No. 031103.

(21) Coenen, T.; Vesseur, E. J. R.; Polman, A. Angle-Resolved Cathodoluminescence Spectroscopy. Appl. Phys. Lett. 2011, 99, 143103.

(22) Coenen, T.; Vesseur, E. J. R.; Polman, A.; Koenderink, A. F. Directional Emission from Plasmonic Yagi-Uda Antennas Probed by Angle-Resolved Cathodoluminescence Spectroscopy. Nano Lett. 2011, $11,3779-3784$.

(23) Suzuki, T.; Yamamoto, N. Cathodoluminescent Spectroscopic Imaging of Surface Plasmon Polaritons in a 1-Dimensional Plasmonic Crystal. Opt. Express 2009, 17, 23664-23671. 
(24) Yamamoto, N.; Ohtani, S.; García De Abajo, F. J. Gap and Mie Plasmons in Individual Silver Nanospheres near a Silver Surface. Nano Lett. 2011, 11, 91-95.

(25) Takeuchi, K.; Yamamoto, N. Visualization of Surface Plasmon Polariton Waves in Two-Dimensional Plasmonic Crystal by Cathodoluminescence. Opt. Express 2011, 19, 12365-12374.

(26) Adamo, G.; Ou, J. Y.; So, J. A.; Jenkins, S. D.; Deaangelis, F.; MacDonald, K. F.; Diafabrizio, E.; Ruostekoski, J.; Zheludev, N. I. Electron-Beam-Driven Collective-Mode Metamaterial Light Source. Phys. Rev. Lett. 2012, 109, 217401.

(27) Rich, D. H.; Tang, Y.; Konkar, A.; Chen, P.; Madhukar, A. Polarized Cathodoluminescence Study of Selectively Grown SelfAssembled InAs/GaAs Quantum Dots. J. Appl. Phys. 1998, 84, 63376344.

(28) Yamamoto, N.; Bhunia, S.; Watanabe, Y. Polarized Cathodoluminescence Study of InP Nanowires by Transmission Electron Microscopy. Appl. Phys. Lett. 2006, 88, 153106.

(29) Lassiter, J. B.; Sobhani, H.; Knight, M. W.; Mielczarek, W. S.; Nordlander, P.; Halas, N. J. Designing and Deconstructing the Fano Lineshape in Plasmonic Nanoclusters. Nano Lett. 2012, 12, 10581062.

(30) Merano, M.; Sonderegger, S.; Crottini, A.; Collin, S.; Renucci, P.; Pelucchi, E.; Malko, A.; Baier, M. H.; Kapon, E.; Deveaud, B.; et al. Probing Carrier Dynamics in Nanostructures by Picosecond Cathodoluminescence. Nature 2005, 438, 479-482.

(31) Tizei, L. H. G.; Kociak, M. Spatially Resolved Quantum NanoOptics of Single Photons Using an Electron Microscope. Phys. Rev. Lett. 2013, 110, 153604.

(32) Fu, X.; Jacopin, G.; Shahmohammadi, M.; Liu, R.; Benameur, M.; Ganière, J. D.; Feng, J.; Guo, W.; Liao, Z. M.; Deveaud, B.; et al. Exciton Drift in Semiconductors under Uniform Strain Gradients: Application to Bent ZnO Microwires. ACS Nano 2014, 8, 3412-3420.

(33) Meuret, S.; Tizei, L. H. G.; Cazimajou, T.; Bourrellier, R.; Chang, H. C.; Treussart, F.; Kociak, M. Photon Bunching in Cathodoluminescence. Phys. Rev. Lett. 2015, 114, 197401.

(34) Coenen, T.; Polman, A. Optical Properties of Single Plasmonic Holes Probed with Local Electron Beam Excitation. ACS Nano 2014, 8, $7350-7358$.

(35) Mignuzzi, S.; Mota, M.; Coenen, T.; Li, Y.; Mihai, A. P.; Petrov, P. K.; Oulton, R. F. M.; Maier, S. A.; Sapienza, R. Energy-Momentum Cathodoluminescence Spectroscopy of Dielectric Nanostructures. ACS Photonics 2018, 5, 1381-1387.

(36) Mohtashami, A.; Coenen, T.; Antoncecchi, A.; Polman, A.; Koenderink, A. F. Nanoscale Excitation Mapping of Plasmonic Patch Antennas. ACS Photonics 2014, 1, 1134-1143.

(37) Taminiau, T. H.; Karaveli, S.; Van Hulst, N. F.; Zia, R. Quantifying the Magnetic Nature of Light Emission. Nat. Commun. 2012, 3, 979.

(38) Schokker, A. H.; Koenderink, A. F. Lasing at the Band Edges of Plasmonic Lattices. Phys. Rev. B: Condens. Matter Mater. Phys. 2014, 90, 155452.

(39) Gaio, M.; Saxena, D.; Bertolotti, J.; Pisignano, D.; Camposeo, A.; Sapienza, R. A Nanophotonic Laser on a Graph. arXiv:1710.06728v3 2017, na.

(40) Lee, S. Y.; Lee, I. M.; Park, J.; Oh, S.; Lee, W.; Kim, K. Y.; Lee, B. Role of Magnetic Induction Currents in Nanoslit Excitation of Surface Plasmon Polaritons. Phys. Rev. Lett. 2012, 108, 213907.

(41) Brucoli, G.; Martín-Moreno, L. Comparative Study of Surface Plasmon Scattering by Shallow Ridges and Grooves. Phys. Rev. B: Condens. Matter Mater. Phys. 2011, 83, No. 045422.

(42) Rotenberg, N.; Krijger, T. L.; Le Feber, B.; Spasenović, M.; De Abajo, F. J. G.; Kuipers, L. Magnetic and Electric Response of Single Subwavelength Holes. Phys. Rev. B: Condens. Matter Mater. Phys. 2013, $88,241408$.

(43) De Abajo, F. J. G. Colloquium: Light Scattering by Particle and Hole Arrays. Rev. Mod. Phys. 2007, 79, 1267-1290.

(44) Langguth, L.; Punj, D.; Wenger, J.; Koenderink, A. F. Plasmonic Band Structure Controls Single-Molecule Fluorescence. ACS Nano 2013, 7, 8840-8848.
(45) Lukosz, W. Light Emission by Magnetic and Electric Dipoles Close to a Plane Dielectric Interface III Radiation Patterns of Dipoles with Arbitrary Orientation. J. Opt. Soc. Am. 1979, 69, 1495. 